Editorial

\title{
Epilepsy pharmacoresistance - where are we now?
}

Volume 4 Issue 6 - 2016

\section{Editorial}

Epilepsy is the most common chronic neurological disorders, estimated to affect more than 60 million people worldwide. In the majority of these patients, seizures can be effectively suppressed with antiepileptic drugs (AEDs). However, in about one-third of patients (estimated to exceed $40 \%$ in some studies) epilepsy remains resistant to drug therapy despite of availability of more than 30 antiepileptic drugs (AEDs) with various mechanism of action. Approximately $20 \%$ of patients with primary generalized epilepsy and up to $60 \%$ of patients who have focal epilepsy develop drug resistance during the course of their condition, which for many is lifelong. ${ }^{1-2}$

Pharmacoresistant epilepsy (PRE) exacts an enormous toll on patients and their families, while the loss of employment potential and cost of medical care has a substantial impact on society. Individuals who fail to respond or respond only partially to AEDs continue to experience incapacitating seizures that lead to neuropsychological, psychiatric, and social impairments thereby reducing quality of life and increasing morbidity and mortality (SUDEP, trauma, drowning, refractory status epilepticus etc.). ${ }^{3}$

Up to now there has been no uniformly accepted definition of PRE. According to proposal of dedicated task force of the International League against Epilepsy in 2010, PRE may be defined as failure of adequate drug trials of two tolerated and appropriately chosen and used AED regimens to achieve seizure freedom, whether as monotherapy or in combination. ${ }^{4}$ The definition is consistent with the clinical observation that if seizure freedom is not achieved with only two trials of appropriate AED regimens, the likelihood of therapeutic success with subsequent regimens declines sharply.

Resective epilepsy surgery could be successful treatment option in some of those patients, while other surgical options such as ablative procedures (stereotactic laser ablation, stereotactic radiosurgery, SEEG guided RF thermocoagulation), neuromodulation (VNS, DBS, RNS), non-conventional pharmacological (verapamil, atorvastatin, neuropeptide Y, adenosine, etc.) and non-pharmacological interventions (dietary therapies, focal cooling, herbal remedies) still represent palliative procedures. ${ }^{5,6}$

Overall, PRE remains a major medical problem for patients, their families and society which are waiting for satisfactory management.

The first step in overcoming this problem should be revising diagnosis and prescribed therapy. Common causes of treatment failure, such as poor compliance, diagnostic errors or inappropriate selection of first-line AEDs (especially in combination therapy; for example combination of two AEDs with similar mechanism of action instead two with different mechanisms of action), should be addressed early on. Importantly, the presence of "false pharmacoresistance" may not be easily recognizable, and this possibility needs to be investigated in any patient presenting with difficult- to-control seizures. In fact, up to $30-40 \%$ of the patients referred to comprehensive epilepsy centres for video-EEG monitoring are found to have pseudoseizures or nonepileptic seizures (NES), mostly psychogenic non-epileptic seizures (PNES). Minority of them (about 10-15\%) also has epileptic seizures

\author{
Silvio Basic, ${ }^{1,2}$ \\ 'Faculty of Medicine, Josip Juraj Strossmayer University of \\ Osijek, Croatia \\ 2Department of Neurology, University Hospital Dubrava, \\ Croatia
}

Correspondence: Silvio Basic, Faculty of Medicine, Josip Juraj Strossmayer University of Osijek, Cara Hadrijana le, 3 I Osijek, Department of Neurology, University Hospital Dubrava, Avenija Gojka Suska 6, I Zagreb, Croatia, Email sbasic@kbd.hr

Received: July 04, 2015 | Published: July 07, 2016

or has had them in the past. Other medical conditions that mimic and are misdiagnosed as epilepsy could be different neurological disorders (Tourette's syndrome, migraine, cerebrovascular disorders, movement disorders etc.), sleep disorders, syncope, heart arrhythmias, metabolic and electrolyte imbalance etc. Gold standard in differentiation between epileptic and NES is prolonged video-EEG monitoring, and in such cases a diagnosis can be made with high accuracy. ${ }^{7}$ However, we should be aware of limitations of scalp EEG recording, especially in recognising frontal lobe seizures or seizures arising from small or depth foci, which sometimes could not be easily visible, for example due to rapid occurring of muscle artefact or other causes.

Although a variety of risk factors have been associated with PRE (e.g., early onset of seizures, number of seizures before treatment, long history of poor seizure control, having more than one type of seizure, cognitive disability, remote symptomatic etiology, history of status epilepticus; structural abnormalities), the relative contributions of these various factors and the underlying biochemical mechanistic issues associated with each of them, remains a mystery.

Epidemiological studies have suggested three different patterns of drug-resistance in epilepsy:

(A) De novo drug resistance: patients who exhibit pharmacoresistance from the time of onset of their very first seizure. In these patients seizures are difficult to control right from the start (pharmacoresistance has been fully developed before the start of AED treatment).

(B) Progressive drug resistance: In these patients seizures are initially well controlled, but then become refractory over time. This pattern may be observed, for instance, in some childhood epilepsies or patients with mesial temporal lobe epilepsies

(C) Waxing and waning resistance: In this case seizures follow a waxing and waning pattern alternating between a remitting 
(pharmacoresponsive) and relapsing (pharmacoresistant) course. Some of these patients, may become seizure-free later on with additional AED trials (intractability is not sustained). ${ }^{1,8}$

These different patterns of drug-resistance in epilepsy strongly suggest existing of several various patomechanisms underlying epilepsy pharmacoresistance. In the past three decades, various potential mechanisms underlying epilepsy pharmacoresistance or factors predicting poor outcome have arisen, among them two major hypotheses which were thoroughly investigated and discussed: the multidrug-transporter hypothesis and the target hypothesis. ${ }^{9}$

The Transporter hypothesis suggests that inadequate penetration of AEDs across the blood-brain barrier (BBB), caused by increased expression or function of efflux transporters such as P-glycoprotein (PGP) and multidrug resistance-associated proteins (MRPs), leads to insufficient drug levels in epileptogenic brain tissue. These transporters are localized to the BBB where their primary physiologic role is to extrude potentially harmful xenobiotic molecules from the brain. However, there has been controversy as to which AEDs are transported by human PGP. The molecular principle of overexpression has not been clarified - whether it is a result of a second-line protection of the blood-brain barrier caused by seizure impairment, or a result of a chronic barrier dysregulation. Reports in literatures suggest that different transporters could play a role in reduced penetration of the AEDs across the BBB, and even more important, some AEDs are not a multi-drug transporters substrates (levetiracetam for example), but patients non-responders to other AEDs are refractory to levetiracetam as well. ${ }^{10,11}$

Evidence for the other hypotheses, including the popular target hypothesis, is currently more limited. A reduced sensitivity, caused by intrinsic or acquired changes in major targets for many of the clinically established AEDs, such as the voltage-gated sodium channel and the GABAA receptor, has been suggested to have a role in AEDresistant chronic human and experimental epilepsy. ${ }^{12}$ This hypothesis could be plausible explanation of the resistance to some AEDs which acts by specific target, but could not explain resistance to all AEDs acting via different targets and by different mechanisms, since a polymorphism in a single molecular target is unlikely to account for pharmacoresistance to all AEDs.

In recent years, several more potential mechanisms have been implicated in epilepsy pharmacoresistance, such as the network hypothesis, the gene variant hypothesis and the intrinsic severity hypothesis.

The network hypothesis proposes that structural brain alterations and/or network changes (for example, hippocampal sclerosis) are involved in resistance to AEDs. ${ }^{13}$ This could only partial explain pharmacoresistance because not all patients with hippocampal sclerosis and other proposed alterations are pharmacoresistant.

The gene variant hypothesis suggests that there is an inherent resistance that is governed by genetic variants of proteins that are involved in the pharmacokinetics and pharmacodynamics of AED activity. However, it is still hard to believe that so many genetic variants necessary to interfere with all potential pharmacokinetics or pharmacodynamics processes could happen in individual in the same time. Similar but more comprehensive is the hypothesis of mechanisms related to AED - development of tolerance and ineffective mechanisms of action. Tolerance is an adaptive response of the body of reduced response to a drug after repeated administration. It develops to some drug effects much more rapidly than to others and depends on the drug and individual, probably genetic factors. Two major types of tolerance are known: pharmacokinetic (metabolic) tolerance which is due to induction of AED-metabolizing enzymes and could be easily to overcome by increasing dosage, and second, pharmacodynamics (functional) tolerance due to "adaptation" of AED targets (e.g. loss of receptor sensitivity, similar to the previously discussed target hypothesis). ${ }^{14,15}$

In contrary to other hypotheses which are focused to specific pharmacoresistance factors, an alternative intrinsic severity hypothesis suggests that increased disease severity leads to drug intractability. It is based on an intriguing epidemiological finding that a higher seizure frequency in the early phase of epilepsy before treatment increases the risk of drug resistance. An essential point of this hypothesis is that common neurobiological factors may underline both epilepsy severity and drug resistance based on concept that seizures that are easily triggered can result in frequent seizures that may be difficult to suppress. The neurobiological or molecular factors underlying this hypothesis are still unknown, but it is speculated that they include changes of targets and alterations of network properties (e.g. via elimination of cellular elements and/or abnormal network plasticity) that leads to unstable neuronal networks, making it difficult to control seizures. ${ }^{11}$ Although this is an attractive hypothesis it also has weak points: despite the fact that high seizure frequency in patients is often associated with drug resistance, a subgroup of patients can become seizure free after a change in medication. Moreover, there are patients who start with only a few seizures and become pharmacoresistant. Finally, there is no consensus at present regarding the most appropriate clinical measures of epilepsy severity.

Failure of all those hypotheses to clarify mechanisms underlying epilepsy pharmacoresistance clearly shows necessity of different approach to this problem. During the past decades pharmaceutical drug development efforts brought us numerous novel AEDs with new mechanisms of action, however, pharmacological treatment success remains almost the same as 40 years ago, around one third of all patients with epilepsy still remain refractory to treatment with conventional pharmacotherapy. Lack of significant progress in epilepsy pharmacotherapy is logical outcome of established but incomprehensive hypothesis of epilepsy as a condition of neuronal dysfunction, in which inadequate neuronal inhibition or excessive neuronal excitation causes recurrent seizures. Consequently, drug development was guided by the principle that excessive neuronal discharges are the cause of the disease.

From this point of view, particularly important could be the until now relatively underestimated hypothesis of the homeostatic bioenergetic network regulation as a potential novel concept to avoid pharmacoresistance in epilepsy. ${ }^{16}$ This hypothesis consider epilepsy as a disorder of complex network dysfunction, involving not only an imbalance of neuronal excitation and inhibition, but also disruption of immunomodulatory functions, dysfunction of the blood brain barrier, altered function of glia, in particular of astrocytes and microglia, as well as more complex epigenetic changes. Recent studies have implicated astrocytes in important physiological roles in the CNS, such as synchronisation of neuronal firing, ion homeostasis, neurotransmitter uptake, glucose metabolism and regulation of the vascular tone. This novel view on astrocytes as communication partners of neurons rather than 'brain glue' has rekindled the question regarding the role of these cells in neurological disorders such as epilepsy. New understanding of astrocytes function as direct communication partners of neurons in dynamic interaction with synapses through uptake of neurotransmitters, receptor-mediated $\mathrm{Ca}^{2+}$ signalling and subsequent gliotransmitter release gave rise to the term tripartite synapse which comprises not only pre- and postsynaptic 
neuronal elements but also the astrocytic process. Indeed, an increasing body of evidence has documented astroglial dysfunction, and even dysregulation of astroglia-specific functions in human and experimental epilepsy, indicating that dysfunctional astrocytes could play a crucial role in epilepsy. This particularly concerns impaired uptake/conversion of glutamate, removal/redistribution of $\mathrm{K}^{+}$and adenosine dysfunction. For example, down regulation of astrocytic glutamate-converting enzyme glutamine synthetase (GS) leads to decreased metabolism of glutamate to glutamine with accumulation of excitatory neurotransmitter glutamate in the cytoplasm of astrocytes and on the other hand,decreased synthesis of neuronal GABA (inhibitory neurotransmitter), partly because glutamine is a precursor for the synthesis of GABA. Both of this can increase brain excitability and lead to seizures. Malfunction of GS, as observed in epileptic tissue, suggested that astrocyte dysfunction is causative of hyperexcitation, neurotoxicity and the generation or spread of seizure activity ${ }^{17}$ Accordingly, dysfunctional astrocytes should be considered as promising targets for new therapeutic strategies.

Atpresent, therefore, we haveno good understanding of mechanisms involved in pharmacoresistance. Current hypotheses could at the best only partial explain mechanism or mechanisms underlying PRE. As a result of the recognized limitations of these hypotheses, it has been therefore suggested that epilepsy research change its perspective and search for alternative mechanisms. For example alterations in network properties may contribute to pharmacoresistance.

In order to overcome obstacles to seizure control and to improve treatment options for complex network disorders, such as epilepsy, drug development efforts should be shifted from current "neurocentric" concept focused mainly on downstream targets of neuronal signalling to the wider concept focused on non-selective upstream regulators and endogenous regulators of brain homeostasis. Only the synergistic modulation of an entire network will enable us to outflank drug resistance, which is still a major hurdle in effective seizure treatment of epilepsy.

\section{Acknowledgments}

None.

\section{Conflicts of interest}

None.

\section{References}

1. Alexopoulos AV. Pharmacoresistant epilepsy: Definition and explanation. Epileptology. 2013;1(1):38-42.
2. Kwan P, Brodie MJ. Early identification of refractory epilepsy. $N$ Engl J Med. 2000;342(5):314-319.

3. Beghi E. Addressing the burden of epilepsy: Many unmet needs. Pharmacol Res. 2016;107:79-84.

4. Kwan P, Arzimanoglou A, Berg AT, et al. Definiition of drug resistant epilepsy: consensus proposal by the ad hoc Task Force of the ILAE Commission on Therapeutic Straregies. Epilepsia. 2010;51(6):1069-1077.

5. Quigg M, Harden C. Minimally invasive techniques for epilepsy surgery: stereotactic radiosurgery and other technologies. J Neurosurg. 2014;121(Suppl):232-240.

6. Sharma AK, Rani E, Waheed A, et al. Pharmacoresistant Epilepsy: A Current Update on Non-Conventional Pharmacological and NonPharmacological Interventions. J Epilepsy Res. 2015;5(1):1-8.

7. Devinsky O, Gazzola D, LaFrance WC. Differentiating between nonepileptic and epileptic seizures. Nat Rev Neurol. 2011;7(4):210-220.

8. Callaghan B, Schlesinger M, Rodemer W, et al. Remission and relapse in a drug-resistant epilepsy population followed prospectively. Epilepsia. 2011;52(3):619-626

9. Schmidt D, Löscher W. New developments in antiepileptic drug resistance: an integrative view. Epilepsy Curr. 2009;9(2):47-52.

10. Löscher W, Potschka H. Role of multidrug transporters in pharmacoresistance to antiepileptic drugs. J Pharmacol Exp Ther. 2002;301(1):7-14

11. Rogawski MA, Johnson MR. Intrinsic severity as a determinant of antiepileptic drug refractoriness. Epilepsy Curr. 2008;8(5):127-130

12. Remy S, Beck $H$. Molecular and cellular mechanisms of pharmacoresistance in epilepsy. Brain. 2006;129(Pt 1):18-35.

13. Abdelmalik P, Avoli M, Carlen P. Intractable epilepsies of the neocortex: basic mechanisms. Adv Neurol. 2006;97:117-127.

14. Löscher W, Schmidt D. Experimental and clinical evidence for loss of effect (tolerance) during prolonged treatment with antiepileptic drugs. Epilepsia. 2006;47(8):1253-1284.

15. Viteva E. Basic cellular and molecular mechanisms of refractory epilepsy: a review of current hypotheses. Molecular \& Cellular Epilepsy. 2014;1:1-7.

16. Boison D, Masino SA, Geiger JD. Homeostatic bioenergetic network regulation - a novel concept to avoid pharmacoresistance in epilepsy. Expert Opin Drug Discov. 2011;6(7):713-724.

17. Halassa MM, Fellin T, Haydon PG. The tripartite synapse: roles for gliotransmission in health and disease. Trends Mol Med. 2007;13(2):54-63. 